\title{
Transmitted light pH optode for small sample volumes
}

\author{
Christian Rogge ${ }^{1}$, Steffen Zinn ${ }^{1}$, Paolo Prosposito ${ }^{2}$, Roberto Francini ${ }^{2}$, and Andreas H. Foitzik ${ }^{1}$ \\ ${ }^{1}$ University of Applied Science Wildau, Department of Engineering and Natural Sciences, \\ 15745 Wildau, Germany \\ ${ }^{2}$ University of Rome Tor Vergata, Department of Industrial Engineering and INSTM, \\ Via del Politecnico 1, 00133 Rome, Italy \\ Correspondence to: Christian Rogge (rogge@th-wildau.de)
}

Received: 30 September 2016 - Revised: 7 July 2017 - Accepted: 2 August 2017 - Published: 16 October 2017

\begin{abstract}
An innovative concept of a low-cost $\mathrm{pH}$ optode with working volumes of less than $150 \mu \mathrm{L}$ is presented. The $\mathrm{pH}$ monitoring is based on the color changing effect of $\mathrm{pH}$ indicators. The optode includes an RGB color sensor patch TCS34725 from Adafruit, a controllable LED and reactor slides and is addressed by a selfwritten LabVIEW ${ }^{\odot}$ software. Utilizing the hue value of the HSV color model, it is possible to analyze the color change of the indicator and estimate the $\mathrm{pH}$ value of the analyzed samples by exploiting sigmoidal fit models. Measurements carried out with phenol red and DMEM (Dulbecco's Modified Eagle's Medium) reported a standard error of calibration in the physiologic $\mathrm{pH}$ range (6.5-7.5) of $\pm 0.04 \mathrm{pH}$ units.
\end{abstract}

\section{Introduction}

A new approach to fermentation-based bioreactors is the constant miniaturization of bioreactors in the framework of personalized medical care or high-throughput screenings. Such small-scale devices pose great challenges for packaging and interconnection technology and more so for sensor technologies in terms of monitoring cell vitality. The continuous monitoring of cell vitality parameters such as $\mathrm{pH}, \mathrm{pO}_{2}$ and $\mathrm{pCO}_{2}$ are most essential (Yacynych et al., 1990; Weigl et al., 1994) for cell cultivation.

Different setups are possible depending on the actual type of application. In the case of $\mathrm{pH}$ sensors, the use of $\mathrm{pH}$ electrodes in combination with reference electrodes such as $\mathrm{Ag} / \mathrm{AgCl}$ are currently the state of the art. However, these electrodes are not suitable for small sample volumes in the microliter range (Kick and Mertig, 2015). In addition, no commercially available pH electrode could be found that is suitable for a determination of $\mathrm{pH}$ within working volumes of less than $1 \mathrm{~mL}$. The normal $\mathrm{pH}$ measurement in laboratories is carried out with $\mathrm{pH}$ indicators (titration) or $\mathrm{pH}$ stripes. These so-called colorimetric methods are very important for the determination of $\mathrm{pH}$ in small sample volumes (Offenbacher et al., 1986). In the past, a lot of effort has been invested in developing novel $\mathrm{pH}$ sensors. In compar- ison to electrochemical $\mathrm{pH}$ sensing, optical sensors offer a good alternative when measuring a small dynamic range of about $2 \mathrm{pH}$ units (Stahl et al., 2006). Moreover, optical sensors seem to have very good properties in terms of stability to electrical interference stability, measurement stability and quality in extreme $\mathrm{pH}$ regions. They offer the possibility of remote sensing and real-time analysis (Safavi and Bagheri, 2003). Further advantages are the lack of a need for a reference element and the possibility of low-cost, autoclavable and precalibrated sensor patches (Capel-Cuevas et al., 2010). In addition to these advantages, developments in electronics, material science and computer technologies as well as the increasing number of inexpensive optical components and the wide range of synthetic reagents for colorimetric systems have pushed research in the field of optical-based $\mathrm{pH}$ sensors enormously (Lau et al., 2004). Besides all these efforts, there is still a high demand for low-cost, selective and realtime monitoring systems to determine $\mathrm{pH}$ values (Liu et al., 2014), which combines all of these attributes.

The measurement principles of optodes are well suited to overcome the challenges of monitoring small sample volumes. According to the current literature in the field of optical $\mathrm{pH}$ sensing, a broad range of applications, measurement techniques and $\mathrm{pH}$ indicators are under development (Lin, 2000). Measurement techniques are mainly focused on 


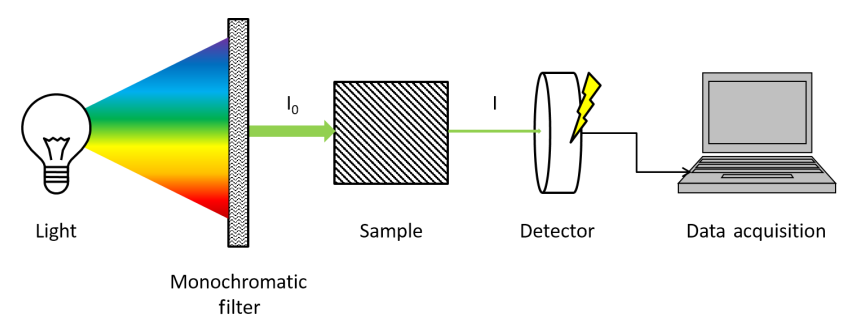

Figure 1. Sketch of the components of a simplified optode concept.

the utilization of UV-VIS spectra, fluorescence lifetimes and colorimetric measurements. On the basis of the individual measurement technique, a special indicating element is chosen such as chemicals or physical phenomena. Some chemicals for example change their color when encountering different $\mathrm{pH}$ values ( $\mathrm{pH}$ indicators) while other substances exhibit fluorescent properties. These chemicals have to come into contact with the sample to give information about the respective $\mathrm{pH}$ value. Therefore, different immobilization techniques are used to specify the area and time of contact between indicating chemicals and the sample itself. Moreover, the immobilization technique and the chosen measurement technique determine the field of use for the developed $\mathrm{pH}$ optodes. The utilization of indicators entrapped in membranes or the use of fiber optics coated with indicating elements are only a small scope of the research done in the field of $\mathrm{pH}$ sensing optodes. A simplified concept of optodes in general is displayed in Fig. 1. The light-emitting source can vary from high-power lasers to specifically colored LEDs or common white LEDs with a very broad spectral range. In most cases, the spectra are sharpened with the aid of monochromatic filters before reaching the sample. Within the sample, specific effects like absorbance (Jin et al., 2000), fluorescence (Song et al., 1997) or phosphorescent emission may occur. These effects are used in many research approaches as sensing elements. Additionally, evanescence-field measurements can also be used for optode concepts (Flannery et al., 1997).

The aim of this research is the development of a nondestructive, contact-free and cost-effective $\mathrm{pH}$ optode for samples with working volumes less than $150 \mu \mathrm{L}$. Therefore, a sensor system based on transmitted light is developed, which can be easily used for micro-structured microfluidic arrangements. The presented $\mathrm{pH}$ optode determines the color change of phenol red $(\mathrm{PhR})$, bromothymol blue (BTB) and Dulbecco's Modified Eagle's Medium (DMEM) depending on different $\mathrm{pH}$ values.

Magnusson et al. (2013) presented a possibility to determine the $\mathrm{pH}$ with nanoliter volumes within a microfluidic cell culture system. They used a standard laboratory microscope combined with a scientific-grade digital camera and two band-pass filters. In comparison to our prototype, they achieved very good signals in a complectly different quantity of working volume but with a more expensive and complex setup.

Brown et al. (2014) reported a pH monitoring system for $100 \mu \mathrm{L}$ samples in a 384-well plate using a digital camera. The captured RGB values of the pictures are converted into HSV or HSL color space. As shown in this paper, an optical approach for the determination of $\mathrm{pH}$ values can be versatile, efficient and provide a new method of application. Nevertheless, a similar yet different approach is reported, which is primarily focused on the use of more cost-effective sensor elements and a smaller footprint size. The use of membranes doped with indicators or fiber optics coated with indicating elements such as those reported by Maclin et al. (2015) are not sufficient for the aimed approach, due to problems in biofouling and cross contamination of the cells with the contact area. Rajan et al. (2016) worked in parallel to this report on a quite similar approach for a non-contact real-time $\mathrm{pH}$ measurement system. In contrary to the used RGB sensor patches, Rajan et al. (2016) established a transmissionbased LED as light source and a specific photodiode to obtain a spectra of a specific wavelength. They were also able to achieve a small footprint with an easy integration in different cell culture systems.

\section{Experimental setup}

PhR (0.04 g, P3532 Sigma-Aldrich) was dissolved in $1.13 \mathrm{~mL}$ of $\mathrm{NaOH}(0.1 \mathrm{M})$ and filled with purified water up to $100 \mathrm{~mL}$. The stock solution of BTB was made by diluting $0.1009 \mathrm{~g}$ of the reagent in $20 \mathrm{~mL}$ of EtOH and filled with deionized $\mathrm{H}_{2} \mathrm{O}$ up to $100 \mathrm{~mL}$.

Measurements were accomplished with sodiumpotassium phosphate buffers (SPPBs) in different concentrations to get a stable array of $\mathrm{pH}$ values ranging from 5 to 8 in $0.1 \mathrm{pH}$ steps. The $\mathrm{pH}$ indicators and the specific SPPBs were mixed in different dilutions. All applied $\mathrm{pH}$ values were observed with a $\mathrm{pH}$ electrode (Mettler Toledo InLab 490) in a $10 \mathrm{~mL}$ solution of each sample. Then, $150 \mu \mathrm{L}$ of each solution was spread into each of the eight micro-bioreactor mock-ups on the polycarbonate slide as shown in Fig. 2.

Furthermore, experimental results using DMEM (Dulbecco's Modified Eagle's Medium, Thermo-Fisher Scientific, catalog number: 11965092) are reported. For these experiments, $\mathrm{pH}$ shifts were achieved by adding $\mathrm{HCl}$ to the DMEM.

\section{Results}

\subsection{Prototype}

For transmittance measurements of $\mathrm{pH}$ dependent color changing effects of $\mathrm{PhR}$ and $\mathrm{BTB}$, the light is directly passing through the sample. Therefore, a light source is placed underneath the sample and the light is collected by the RGB sensor 


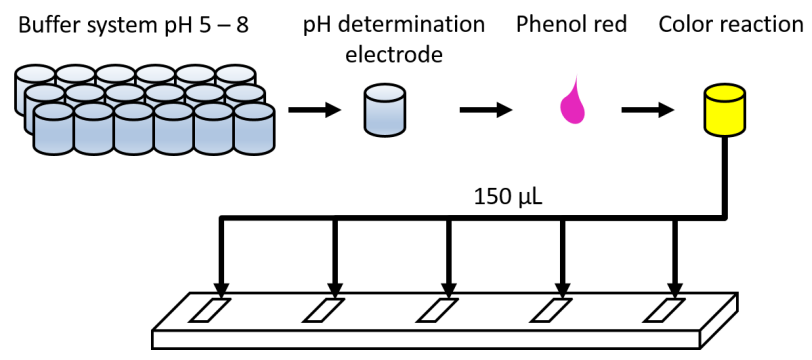

Figure 2. Diagram of the experimental procedure.

patch. The idea was initially visualized in a CAD sketch as shown in Fig. 3, and later manufactured by rapid prototyping via micro-milling and 3-D printing. This approach leads to a very cost-effective measurement system, which in general depends only on the RGB sensor and a control board, e.g., an Arduino Uno board. The expenses for both of these parts is in total EUR 30. Further costs only arise depending on the framework used around these two parts. For this prototype, the overall costs were less than EUR 50.

The manufactured prototype is shown in Fig. 4. Within the figure a simple sketch is included, which illustrates the working principle and the adaption of this prototype in comparison to the common optode concepts shown in Fig. 1. As mentioned before, optodes usually contain modified fiber optics or membrane technologies and monochromatic filters. Within this prototype none of these techniques are required. Instead of changing the geometrical dimension of the microbioreactors to implement membranes or fiber optics, the $\mathrm{pH}$ indicators are already included in the nutrition media and thus can be utilized to measure the $\mathrm{pH}$. Secondly, no additional monochromatic filter is necessary since the RGB unit has chromatic filters on top of the respective photodiodes.

The LED originally mounted close to the top of the RGB detection unit (Fig. 4a) was desoldered, incorporated underneath the sample holder as indicated in Fig. $4 \mathrm{c}$ and reconnected electrically with the RGB sensor via small wires. Data acquisition and the control of the light source was achieved by an Arduino ${ }^{\odot}$ micro-controller. The user interface and the data storage were created using a LabVIEW $^{\odot}$ program.

The basic idea behind the colorimetric measurement with the RGB sensor is shown in Fig. 5 for the example of PhR. In acidic $\mathrm{pH}$ regions $(<6) \mathrm{PhR}$ indicates the $\mathrm{pH}$ through a yellow color. Following chromatic rules, high signals of green, less of red and least of blue are expected. In near-neutral $\mathrm{pH}$ regions, PhR changes the color from yellow to orange because the amount of protonated PhR increases. Thus, the RGB unit should measure decreasing signals of green and increasing signals of red and blue. With even higher $\mathrm{pH}$ values of more than 7.3 the protonated form of PhR dominates and gives the solution a magenta shade with a tendency towards red. The signals detected should change to high amounts of red and blue and very low amounts of green. In consideration

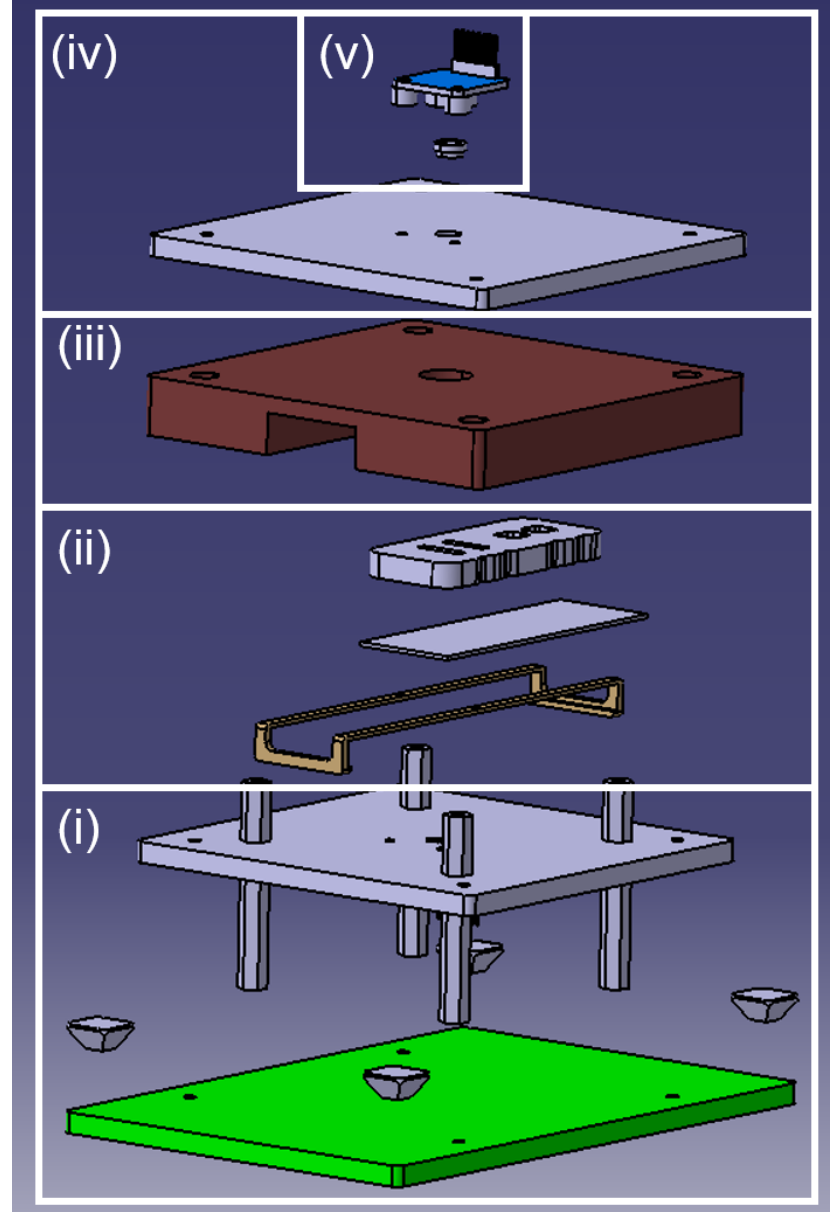

Figure 3. CAD explosion sketch of the optode: (i) base layer for better handling and a solid positioning, (ii) reactor mock-up placed on top of the slide, (iii) placeholder for reactor mock-ups with different thicknesses, (iv) top layer and (v) RGB sensor and an incorporated lens.
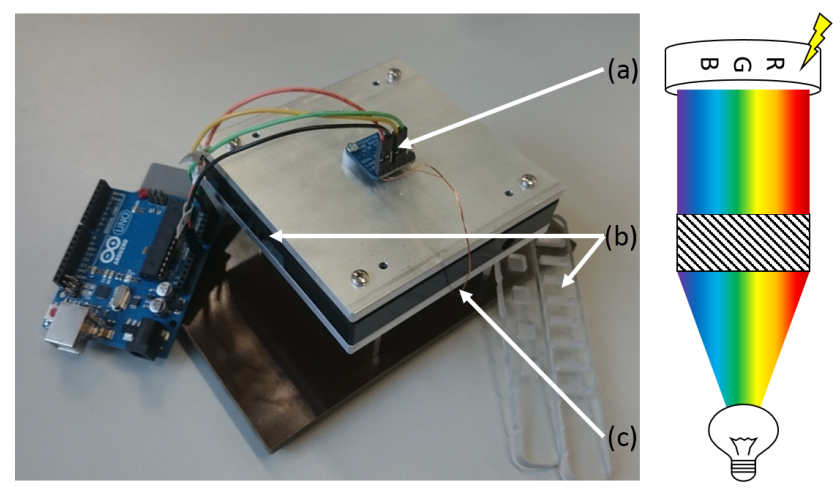

Figure 4. Picture of the prototype for transmitted light measurements consisting of (a) RGB detection unit, (b) a sample holder and (c) a LED. The diagram on the right shows the measurement principle. 

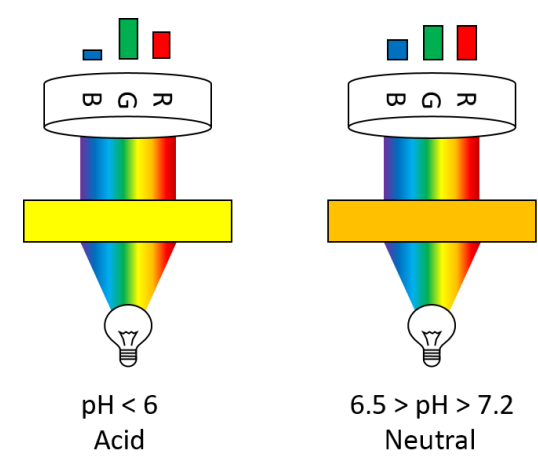

$6.5>\mathrm{pH}>7.2$

Neutral

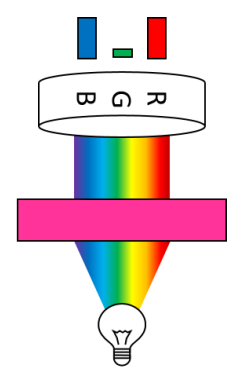

$\mathrm{pH}>7.3$

Alkaline

Figure 5. Diagram for the measurement principle of the transmitted light prototype with the color changing effect of $\mathrm{PhR}$ and the corresponding red, green and blue signals.

Table 1. Estimated color angles of BTB (bromothymol blue) and $\mathrm{PhR}$ (phenol red) in different $\mathrm{pH}$ values based on the HSV color space model with hue values in brackets indicating the corresponding negative hue value.

\begin{tabular}{rlrlr}
\hline $\mathrm{pH}$ & PhR color & $\begin{array}{r}\text { PhR hue } \\
\left({ }^{\circ}\right)\end{array}$ & BTB color & $\begin{array}{r}\text { BTB hue } \\
\left({ }^{\circ}\right)\end{array}$ \\
\hline 4 & yellow/red & 60 & yellow/green & 70 \\
$7-8$ & orange & 30 & green/cyan & 140 \\
10 & magenta & $300(-60)$ & blue & 240 \\
\hline
\end{tabular}

of the explained example, the transformed hue values for $\mathrm{PhR}$ and additionally for BTB are summarized in Table 1.

Different measurements with various concentrations of $\mathrm{PhR}$ and BTB were carried out. BTB was used as a control chemical for evaluation purposes. Furthermore, experiments containing nutrition media were carried out and will be presented in Sect. 3.6.

The following data show sigmoidal shaped functions, which is a result of the color change of indicators. This can be either related to changes of the $\mathrm{pH}$ value (Capel-Cuevas et al., 2010) or to concentrations of specific analytes (Hong and Chang, 2014). In general, the data fitting can be achieved with different sigmoidal models such as the Boltzmann equation, the logistic equation or the Hill function. However, within this work the fittings were achieved with the doseresponse equation utilized by programs like Origin or R. A modified dose-response equation containing a hill slope coefficient showed the best fitting results regarding the least rootsquare sum and the double standard deviation $(2 \sigma)$.

Two different models were used to describe the sigmoidal shaped function of PhR (Eq. 1) and BTB (Eq. 2).

$$
\begin{aligned}
& H_{\text {Sig }}=H_{\min }+\frac{H_{\max }-H_{\min }}{1+10^{\left(\mathrm{pH}_{\mathrm{pK}}\right) \alpha}} \\
& H_{\text {Sig }}=H_{\min }+\frac{H_{\max }-H_{\min }}{1+10^{\left(\mathrm{pK}_{a}-\mathrm{pH}\right) \alpha}}
\end{aligned}
$$

$H_{\max }$ and $H_{\min }$ are the respective hue values for the top and bottom plateau, $H_{\text {Sig }}$ is the measured hue value given for a specific $\mathrm{pH}$ value, $\alpha$ is used to describe the slope steepness (hill coefficient) and $\mathrm{pK}_{a}$ is the logarithmic acid dissociation constant of the indicator used.

On the basis of this mathematical approach, the calibration data, and thus the $\mathrm{pH}$ value, depend on four different parameters, which have to be fitted first. $H_{\max }$ and $H_{\min }$ should be determined experimentally at very high and low $\mathrm{pH}$ values because they indicate the maximum of the protonated and deprotonated form of the indicator used. It is said that most common indicators reach their respective maximum and minimum at about $\mathrm{pH}=\mathrm{pK}_{\mathrm{a}} \pm 3$ (Jander, 1933; Tucker et al., 1989; Chen et al., 2010). Therefore, all experiments were carried out in a $\mathrm{pH}$ range from 4 to 10 to fulfill these requirements for both $\mathrm{PhR}$ and BTB.

Although the $\mathrm{pK}_{\mathrm{a}}$ is commonly said to be constant, it depends on temperature, ionic strength and the solvent dielectric constant (Reijenga et al., 2013). PhR changes its color from yellow to magenta/red in the $\mathrm{pH}$ range between 6.4 and 8.2 with a $\mathrm{pK}_{a}=7.4-7.9$ (Robert-Baldo et al., 1985; Salk et al., 1954). The color change of BTB appears at the limits of $\mathrm{pH}$ in between approximately 5.8 and 7.6 with a $\mathrm{pK}_{a}=7.1$ (Jeffery et al., 1991; Dean, 1992). Taking this into account, the $\mathrm{pK}_{\mathrm{a}}$ value is one of two parameters needed to be fitted mathematically. The second parameter is the so-called hill slope coefficient $\alpha$ (often referred as $H$, Di Veroli et al., 2015, or $d$, Gadagkar and Call, 2015).

Considering the different approaches of $\mathrm{pK}_{\mathrm{a}}$ and $\alpha$, these parameters have to be fitted within a calibration. Thus, the mathematical model is fitted on the experimental data based on the method of the least root-square sum without weighting as shown in Eq. (4):

$$
\begin{aligned}
\text { Distance } & =\sum\left(\mathrm{Y}_{\text {Data }}-\mathrm{Y}_{\text {Curve }}\right)^{2}, \\
\mathrm{RSS} & =\sqrt{\text { Distance }},
\end{aligned}
$$

where RSS is the root-square sum, $Y_{\text {Data }}$ is the measured hue value and $Y_{\text {Curve }}$ is the hue value calculated with the model (Motulsky and Christopoulos, 2004). The fitting itself is done by the solver function of MS Excel using the GRG-solver algorithm. This algorithm changes the $\mathrm{pK}_{\mathrm{a}}$ and $\alpha$ values starting at $\mathrm{pK}_{\mathrm{a}}=7.1$ and $\alpha=1$ to minimize the RSS value. As long as the $\mathrm{pK}_{\mathrm{a}}$ is within a range of 7 to 8 and $\alpha$ is within a range between 0.85 and 1.5 , the data were determined to be fitted well. 


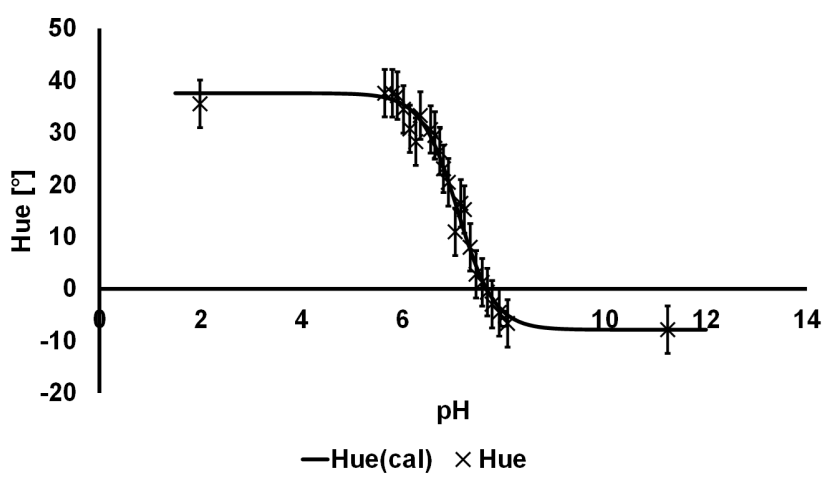

Figure 6. Hue data (mean value of 10 measurements) depending on $\mathrm{pH}$ values. $\mathrm{PhR}$ diluted in a ratio of $1: 10$ in SPPB. The fitting parameters are the following: $\mathrm{pK}_{\mathrm{a}}=7.13, \alpha=1.20$ and $2 \sigma=4.55^{\circ}$.

\subsection{Phenol red (PhR) 1:10 in SPPB}

Regarding the data shown in Fig. 6, the measured color angle of $\mathrm{PhR}$ ranges from $H \sim 40^{\circ}$ at $\mathrm{pH}=2$ to $H \sim-7^{\circ}$ at $\mathrm{pH}=11$. Therefore, $\mathrm{PhR}$ should be yellow with a slightly red shift in alkaline $\mathrm{pH}$ regions, whereas it should be red with a tendency to magenta in acidic $\mathrm{pH}$ regions. Observations with the naked eye estimated hue values between $60^{\circ}$ (yellow) and $-40^{\circ}$ (magenta, slightly red shifted). Although these data display a discrepancy between estimated (shown in Table 1) and measured signals, the fitting results show stable measurement results. However, in acidic $\mathrm{pH}$ regions the discrepancy is quiet significant. The hue value in basic $\mathrm{pH}$ regions implies a strong red color, whereas the observable color contains much more magenta. This effect can be explained with an existing effect of the LED on the measurement. Therefore, two different experiments were carried out to validate this effect. First the concentration of PhR was increased to achieve a higher interaction between the dye and the light. Secondly, the hue of the LED without any dye included was determined.

\subsection{Phenol red 1:5 in SPPB}

A PhR : SPPB ratio of $1: 5$ was chosen to be measured using the developed prototype. The results, in the form of hue values, are shown in Fig. 7. The included error bars indicate the double standard deviation of the distance between the measured hue and the fitted data point. Within these results a double standard deviation of $1.5^{\circ}$ was achieved. In comparison to the data shown before, a higher accuracy was obtained with a higher concentration of the indicator. This is basically induced by the stronger color which enables a higher level of light-matter interaction. An increase in excited indicator molecules consequently results in more distinct hue values. Additionally, the influence of the LED is decreased, because reduced non-interacted light emitted by the LED shines through the sample. Although the $\mathrm{H}_{\max }$ value obtained

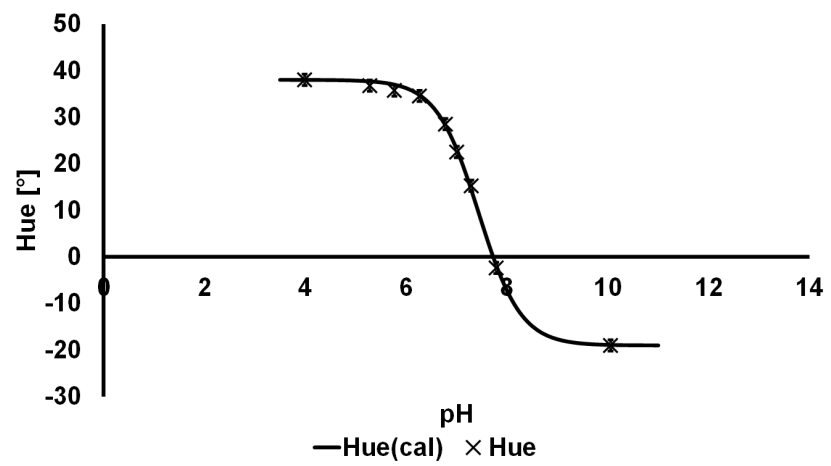

Figure 7. Hue data (mean value of 10 measurements) depending on $\mathrm{pH}$ values measured in a single chamber. $\mathrm{PhR}$ diluted in a ratio of $1: 5$ in SPPB. The fitting parameters are the following: $\mathrm{pK}_{\mathrm{a}}=7.43$, $\alpha=1.04$ and $2 \sigma=1.21^{\circ}$.

is nearly the same, $H_{\min }$ is different with $H_{\min }=-19.02^{\circ}$. This hue value still displays a discrepancy to the expected hue value of about $-40^{\circ}$, but shows a far better tendency towards magenta. Considering this information, a change of the concentration of $\mathrm{PhR}$ did not result in a change of the maximum hue value, but resulted in a change of the obtained minimum.

\subsection{Influence of the LED}

Different hue measurements of PhR imply a possible influence of the LED on these measurements. For evaluation purposes, the color angle of the used LED was obtained and compared with hue values measured in two different $\mathrm{PhR}$ concentrations. The results of this experiment are reported in Fig. 8. In fact, the hue value of the $\operatorname{LED}\left(H=34.33^{\circ}\right)$ and the hue values of $\mathrm{PhR}$ in very acidic $\mathrm{pH}$ regions $\left(H_{\max }(1: 10)=\right.$ $37.57^{\circ}$ and $H_{\max }(1: 5)=38.04^{\circ}$ ) are very similar to each other. Currently, the results seem to indicate that the difference within acidic $\mathrm{pH}$ regions is not significantly influenced by a higher concentration of $\mathrm{PhR}$. In contrast to the acidic $\mathrm{pH}$ regions, the difference between the color of the LED and the color of $\mathrm{PhR}$ in alkaline $\mathrm{pH}$ regions $\left(H_{\min }(1: 10)=-7.83^{\circ}\right.$ and $\left.H_{\min }(1: 5)=-19.02^{\circ}\right)$ is much higher, and the hue values of the two PhR concentrations vary from each other. PhR has an absorbance maximum in the wavelength region of yellow light between 550 and $580 \mathrm{~nm}$. With increasing $\mathrm{pH}$ values, more yellow light of the LED is absorbed, which results in the color change from yellow to magenta. Due to this absorption characteristic of $\mathrm{PhR}$, the difference of the hue value between the LED and $\mathrm{PhR}$ in acidic $\mathrm{pH}$ regions is not influenced by the concentration of the dye. However, the effect of the absorbance of yellow light is much higher within alkaline $\mathrm{pH}$ regions and therefore dependent on the concentration.

In addition to the effect caused by the color spectrum of the LED, the position of the LED with respect to the sample is said to have a high influence on the measurement results. 


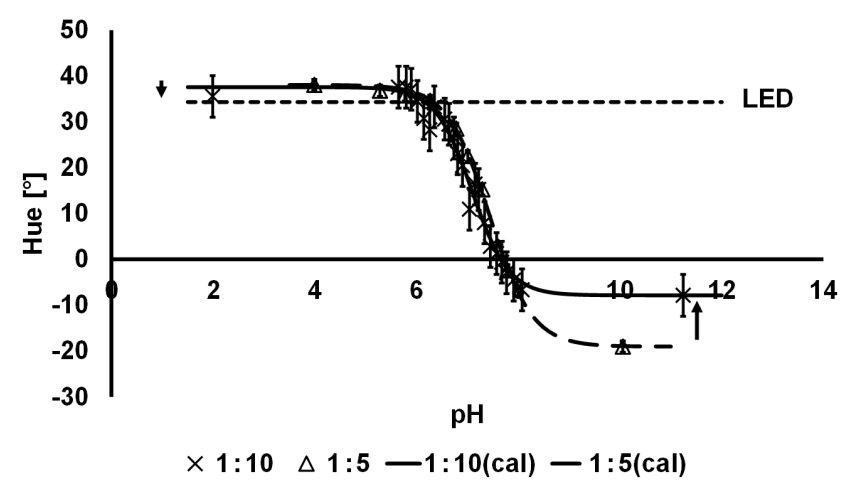

Figure 8. Direct comparison of the results of $\mathrm{PhR}: \mathrm{SPPB}$ with ratios $1: 10$ and $1: 5$. Hue value of the LED and the probable influence of the LED on the results are indicated by the black arrow.

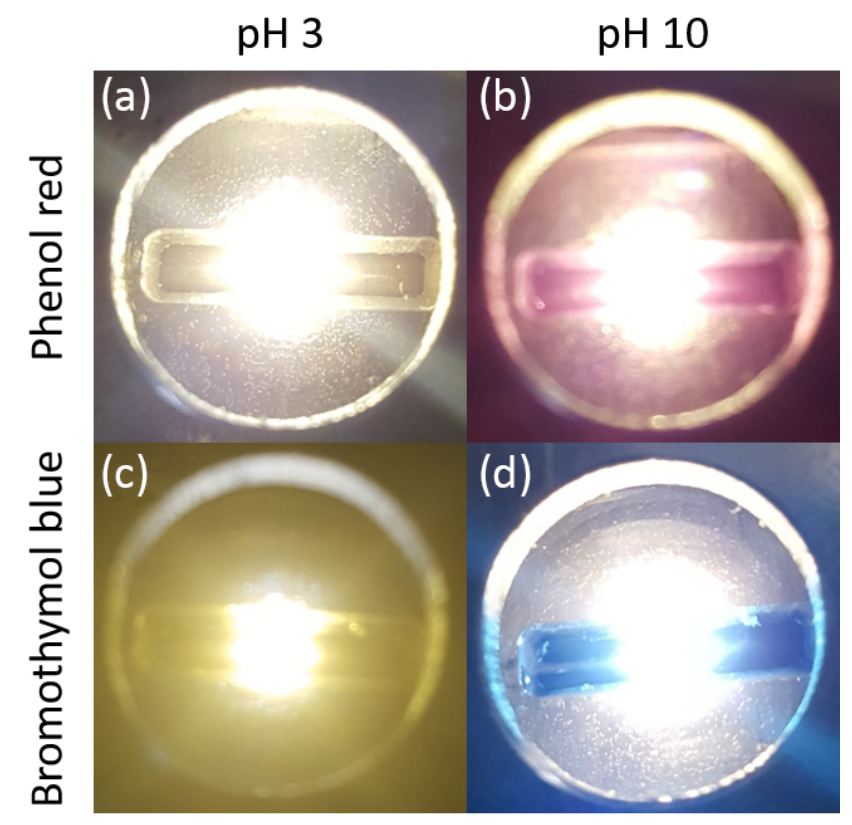

Figure 9. Pictures of the LED shining through the reactor mock-up filled with $\mathrm{PhR}(\mathbf{a}, \mathbf{b})$ and BTB (c, d) in pH 3 (Left column) and $\mathrm{pH} 10$ (right column). Pictures were taken from above while the RGB sensor of prototype II was dismounted.

A matrix of pictures of $\mathrm{PhR}$ and BTB $(1: 10)$ at $\mathrm{pH} 3$ and $\mathrm{pH} 10$ was made and is shown in Fig. 9. To achieve this, the top layer of the second prototype was dismounted. From the edge to the center of the chamber, the intensity of the LED becomes stronger. This is mainly caused by the semicircular angle of radiation of the LED, thus yielding different path lengths and reflection angles of the light, which result in a specific light cone. The nearly unaffected light of the LED takes up about one-third of the complete chamber. Despite that, the experimental results show stable and reproducible signal sequences.

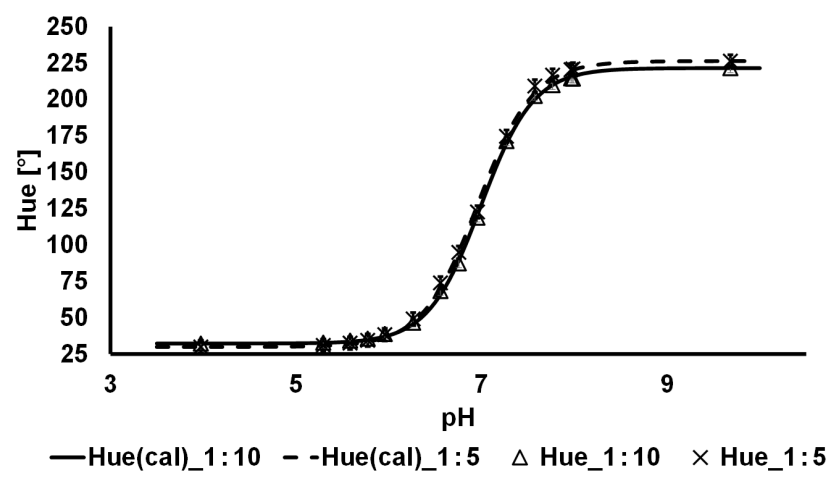

Figure 10. Hue data (mean value of 10 measurements) depending on $\mathrm{pH}$ values measured in the same chamber. BTB diluted in ratios of $1: 10$ and $1: 5$ in SPPB. Fitting parameters are the following: $\mathrm{pK}_{\mathrm{a}}(1: 10)=6.99, \alpha(1: 10)=1.53,2 \sigma(1: 10)=2.97^{\circ}$, $\mathrm{pK}_{\mathrm{a}}(1: 5)=6.96, \alpha(1: 5)=1.46$ and $2 \sigma(1: 5)=4.32^{\circ}$.

\subsection{Bromothymol blue (BTB)}

For a better comparison of the effect of different concentrations of BTB in SPPB, the results for two different dilution ratios are reported within one diagram shown in Fig. 10. Both curves for $H(1: 10)$ and $H(1: 5)$ share almost the same lower limit of $H_{\min }(1: 10)=32.24^{\circ}$ and $H_{\min }(1: 5)=29.93^{\circ}$. Additionally, the maximum plateaus with $H_{\max }(1: 10)=221.51^{\circ}$ and $H_{\max }(1: 5)=226.38^{\circ}$ are also nearly the same. The main parts of the curves are mostly identical, although a small difference can be seen.

Conversely to the initial assumption and comparing these results with the results of different concentrations of $\mathrm{PhR}$, no differences between the two used concentrations of BTB were obtained. One possible explanation could be that both of the utilized BTB concentrations already show the maximum of the obtainable signals. In conclusion, BTB can be diluted even further in comparison to PhR because even with a dilution ratio of $1: 10$ the solution is still saturated with BTB. Therefore, BTB is more suitable for $\mathrm{pH}$ monitoring in even smaller micro-reactors in comparison to $\mathrm{PhR}$. Only very small deviations are detected for the two dilutions of $1: 10$ and $1: 5$ in Fig. 10. These minor deviations are due to the mechanical manufacturing of the chambers resulting in slightly different surfaces (flatness, roughness, micro-scratches etc.) and the subsequent different optical properties of the chambers (transparency, scattering etc.). In the case of industrial manufacturing routines, even such small differences may be eliminated resulting in very precise determinations for the $\mathrm{pH}$ values.

\subsection{Nutrition media}

Results reported beforehand were aiming for a characterization of PhR and BTB in pH ranging from about 3 to 10 . Nevertheless, the following section is more focused on a defined $\mathrm{pH}$ range from $\mathrm{pH}=4$ to $\mathrm{pH}=7.5$. During cell culture, 
it is very uncommon for the solution to become alkaline. In most cases, dying cells produce specific acids which change the $\mathrm{pH}$ value of the nutrition media towards lower $\mathrm{pH}$. Taking this into account, the non-diluted DMEM nutrition media with a basis $\mathrm{pH}$ of about 7.5 is used as the starting point. By using $1 \mathrm{M} \mathrm{HCl}$, the $\mathrm{pH}$ values were set to acidic $\mathrm{pH}$ ranges. But conversely to pure $\mathrm{PhR}$ and $\mathrm{BTB}$, the nutrition media is a far more complex solution. Besides different salts, liquids and nutrients such as glucose, it contains a $\mathrm{pH}$ buffer system to stabilize the $\mathrm{pH}$ in certain ranges for a better cell growth. The DMEM used has a sodium bicarbonate buffer included. This buffer system leads to a rising bubble formation within the solution at low $\mathrm{pH}$ values. These bubbles are caused by $\mathrm{CO}_{2}$, which is produced during the following chemical reaction:

$\mathrm{NaHCO}_{3}+\mathrm{HCl} \rightarrow \mathrm{NaCl}+\mathrm{CO}_{2}+\mathrm{H}_{2} \mathrm{O}$.

A good example for this chemical reaction can be found within the human digestive system. However, within very small working volumes of $100-150 \mu \mathrm{L}$, these bubbles can have a high influence on the measured hue value because of additional scattering effects and local changes of the dye concentration.

The hue data of four different chambers are reported in Fig. 11. The curve has to be read from the near-neutral $\mathrm{pH}$ value backwards to the acidic $\mathrm{pH}$ range. With each experimental step, more $\mathrm{HCl}$ was added and therefore, the $\mathrm{pH}$ value shifted from 7.5 to 4 . It was not possible to obtain any kind of usable data if nutrition media and $\mathrm{HCl}$ were pipetted together within the $150 \mu \mathrm{L}$ chambers. The $\mathrm{CO}_{2}$ bubbles developing within the chamber had a strong negative influence on the measurements. Therefore, the $\mathrm{pH}$ was set in a beaker glass containing the stock solution of the nutrition media by adding small amounts of $\mathrm{HCl}$. The developing bubbles were stirred out within this beaker glass. Meanwhile, the $\mathrm{pH}$ value is controlled with a $\mathrm{pH}$ glass electrode. After specific $\mathrm{pH}$ values were reached, the solution was taken from the beaker glass into specific reactor mock-up chambers. Although most of the evolving gas was stirred out, it was not possible to release all of the bubbles. The remaining $\mathrm{CO}_{2}$ bubbles were expected to have a high influence. However, no significant additional scattering was detected.

Although just small amounts of $\mathrm{PhR}$ are included within the used DMEM, the reported data display a high accuracy and stability within the hue value. The color change of PhR can be detected as a function of the $\mathrm{pH}$.

\section{Conclusions}

This prototype was designed and manufactured as a transmission setup using an RGB color sensor patch to observe the color change of specific $\mathrm{pH}$ indicators. While the RGB data already achieved good results, an even greater improvement was obtained by utilizing the HSV color space. Therefore,

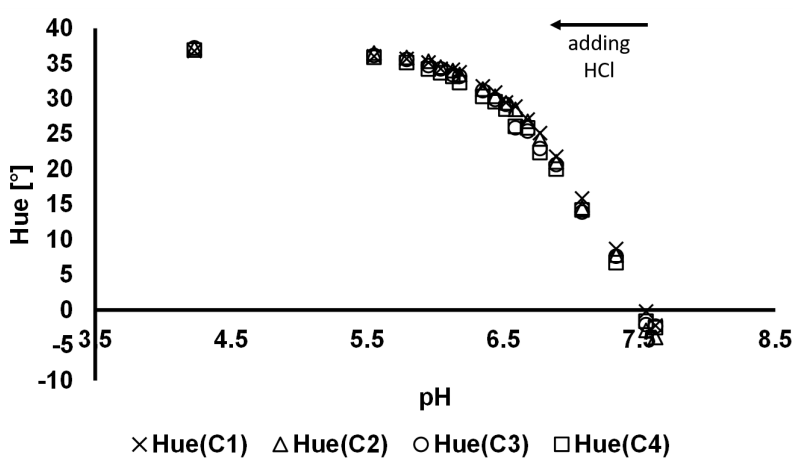

Figure 11. Hue data (mean value of 10 measurements) of nondiluted nutrition media as a function of $\mathrm{pH}$ values measured in four different chambers at once.

the red, green and blue signals were transformed into the hue value, which had two major benefits. On the one hand, the hue value is a perception-based color model and simplifies the signal estimation. Therefore, it is easier to compare the measured data with the real data. On the other hand, the hue value showed better results in terms of stability and accuracy. The accuracy was measured with the double standard deviation of the differences between fitted curve and real measurement data. This very robust fitting procedure, which used $H_{\max }$ and $H_{\min }$, the $\mathrm{pK}_{\mathrm{a}}$ value and the $\alpha$ coefficient, can be adapted for different $\mathrm{pH}$ indicator dyes. The values for $H_{\max }$ and $H_{\mathrm{min}}$ are determined experimentally, while $\mathrm{pK}_{\mathrm{a}}$ and $\alpha$ are fitted mathematically.

The validation of this prototype included two $\mathrm{pH}$ indicator dyes. These were analyzed under different conditions, such as changes in concentration. PhR was used with two different concentrations: $113 \mu \mathrm{M}(1: 10)$ and $226 \mu \mathrm{M}(1: 5)$.

Further validation experiments were carried out using BTB with concentrations of $161 \mu \mathrm{M}(1: 10)$ and $322 \mu \mathrm{M}$ (1:5). Conversely to PhR, BTB changes the color from yellow (acidic) to blue (alkaline) and did not indicate a concentration dependency on the hue measurement. A possible explanation was found in the saturation of the solution with BTB.

Although measurements of low $\mathrm{PhR}$ concentrations (11.3 $\mu \mathrm{M}$, not reported) did not result in obtainable color changes, experiments with DMEM including similar PhR concentrations between 20 and $40 \mu \mathrm{M}$ were successful. DMEM is a very complex solution incorporating amino acids, inorganic salts and many other nutrients. These additional components increase the amount of scattered light significantly. A higher amount of light-matter interaction occurs due to this additional scattering within the solution. Therefore, more PhR molecules are excited and thus more light is absorbed.

Further experiments revealed two major shortcomings when using the second prototype to determine the $\mathrm{pH}$ in DMEM. On the one hand, DMEM includes a sodium bicar- 
bonate buffer to stabilize the cell culture, which induces the building of $\mathrm{CO}_{2}$ with decreasing $\mathrm{pH}$ values. These $\mathrm{CO}_{2}$ bubbles have a negative influence on the hue determination. Considering the use of this system within a flow-through cell culture system for micro-sized bioreactors, $\mathrm{CO}_{2}$ bubbles are not a relevant factor. Even if the formation of bubbles takes place, they will be flushed out by the liquid flow. A second negative aspect of using DMEM is based on missing data for basic (alkaline) $\mathrm{pH}$ regions. The fitting procedure has the necessity for $H_{\max }$ and $H_{\min }$, which have to be determined experimentally by using $\mathrm{pH}$ calibrated standards. Normally, cells in the study of necrosis or apoptosis produce specific acids, which lower the $\mathrm{pH}$. It is very uncommon that nutrition media during a cell culture becomes basic. Because of the imprecisely determined $H_{\min }$ value, the fitting of the data becomes more complex and more imprecise. However, the overall system was designed as a cost-effective easy-to-handle $\mathrm{pH}$ monitoring system to determine the vitality of the cells. Therefore, no high-precision analysis of the $\mathrm{pH}$ is required and the system can be used perfectly for this approach.

Considering all experimental results, this prototype showed remarkable properties in terms of signal stability and accuracy; moreover, it offers the possibility for online measurements. In consideration of the later use of the microbioreactor system, the prototype was designed to fit within micro-sized flow-through cell culture systems. Therefore, disadvantages such as bubble formation or missing $H_{\text {min }}$ baselines have to be taken into account in the experimental environment but are less important in the real use. The achieved advantages of this $\mathrm{pH}$ optode can be found within the easy-to-handle and very cost-effective approach. The system can be miniaturized and incorporated into the microbioreactor system with less effort. Furthermore, the calibration of the complete setup can be made for any type of $\mathrm{pH}$ indicator dye, enhancing the usability of the system for a wide range of cell culture experiments. Considering the desired precision of the $\mathrm{pH}$ analysis, it is possible to enhance the accuracy of the measurement data by using light sources with specific wavelengths, incorporating monochromatic filters and with further improvement of the calibration standard.

Data availability. Data are available upon request by contacting the author Christian Rogge (rogge@th-wildau.de).

Competing interests. The authors declare that they have no conflict of interest.

Special issue statement. This article is part of the special issue "Sensors and Measurement Systems 2016". It is a result of the 18th GMA/ITG Fachtagung Sensoren und Messsysteme 2016, Nuremberg, Germany, 10-11 May 2016.
Acknowledgements. We thank Analytical Control Instruments $\mathrm{GmbH}$ for the productive cooperation in the ZIM Cooperation project KF2014167AK4. This project was financially supported in the framework of the Central Innovation Programme for SME by the AiF Projekt GmbH under the auspices of the Federal Ministry for Economic Affairs and Energy (BMWi).

Edited by: Albert Romano-Rodriguez

Reviewed by: three anonymous referees

\section{References}

Brown, J. D., Bell, N., Li, V., and Cantrell, K.: Quantitative pH assessment of small-volume samples using a universal $\mathrm{pH}$ indicator, Anal. Biochem., 462, 29-31, 2014.

Capel-Cuevas, S., Cuéllar, M., de Orbe-Payá, I., Pegalajar, M., and Capitán-Vallvey, L.: Full-range optical pH sensor based on imaging techniques, Anal. Chim. Acta, 681, 71-81, 2010.

Chen, H.-X., Wang, X.-D., Song, X.H., Zhou, T.-Y., Jiang, Y.-Q., and Chen, $\mathrm{X}$.: Colorimetric optical $\mathrm{pH}$ sensor production using a dual-color system, Sensor. Actuat. B-Chem., 146, 278-282, 2010.

Dean, J.: Lange's Handbook of Chemistry, McGraw-Hill, 14th Edn., chap. 8, 8.115-8.116, 1992.

Di Veroli, G. Y., Fornari, C., Goldlust, I., Mills, G., Koh, S. B., Bramhall, J. L., Richards, F. M., and Jodrell, D. I.: An automated fitting procedure and software for dose-response curves with multiphasic features, Sci. Rep.-UK, 5, 14701, https://doi.org/10.1038/srep14701, 2015.

Flannery, D., James, S. W., Tatam, R. P., and Ashwell, G. J.: pH sensor using Langmuir-Blodgett overlays on polished optical fibers, Opt. Lett., 22, 567-569, 1997.

Gadagkar, S. R. and Call, G. B.: Computational tools for fitting the Hill equation to dose-response curves, J. Pharmacol. Toxicol., 71, 68-76, 2015.

Jeffery, G. H., Bassett J., Mendham, J., and Denney, R. C.: Vogel's Textbook of Quantitative Chemical Analysis, Wiley, 668-670, 1991.

Hong, J. I. and Chang, B.-Y.: Development of the smartphonebased colorimetry for multi-analyte sensing arrays, Lab Chip, 14, 1725-1732, 2014.

Jander, G.: Die colorimetrische und potentiometrische PHBestimmung, Angew. Chem.-Ger. Edit., 46, 83-83, 1933.

Jin, Z., Su, Y., and Duan, Y.: An improved optical pH sensor based on polyaniline, Sensor. Actuat. B-Chem., 71, 118-122, 2000.

Kick, A. and Mertig, M.: Characterization of $\mathrm{pH}$-sensitive polymer layers by surface plasmon resonance and quartz crystal microbalance, Phys. Status Solidi A, 212, 1249-1253, 2015.

Lau, K. T., Baldwin, S., Shepherd, R. L., Dietz, P. H., Yerzunis, W. S., and Diamond, D.: Novel fused-LEDs devices as optical sensors for colorimetric analysis, Talanta, 63, 167-173, 2004.

Lin, J.: Recent development and applications of optical and fiberoptic pH sensors, TRAC-Trend Anal. Chem., 19, 541-552, 2000.

Liu, L., Guo, P., Chai, L., Shi, Q., Xu, B., Yuan, J., Wang, X., Shi, X., and Zhang, W.: Fluorescent and colorimetric detection of $\mathrm{pH}$ by a rhodamine-based probe, Sensor. Actuat. B-Chem., 194, 498-502, 2014.

Maclin, A. Q., Kim, M. D., Dergunov, S. A., Pinkhassik, E., and Lindner, E.: Small-Volume pH Sensing with a Capillary Optode 
Utilizing Dye-Loaded Porous Nanocapsules in a Hydrogel Matrix, Electroanalysis, 27, 733-744, 2015.

Magnusson, E. B., Halldorsson, S., Fleming, R. M., and Leosson, K.: Real-time optical $\mathrm{pH}$ measurement in a standard microfluidic cell culture system, Biomed. Opt. Express, 4, 1749-1758, 2013.

Motulsky, H. and Christopoulos, A.: Fitting models to biological data using linear and nonlinear regression: a practical guide to curve fitting, OUP USA, 2004.

Offenbacher, H., Wolfbeis, O. S., and Fürlinger, E.: Fluorescence optical sensors for continuous determination of near-neutral $\mathrm{pH}$ values, Sensor. Actuat., 9, 73-84, 1986.

Rajan, D. K., Patrikoski, M., Verho, J., Sivula, J., Ihalainen, H., Miettinen, S., and Lekkala, J.: Optical non-contact $\mathrm{pH}$ measurement in cell culture with sterilizable, modular parts, Talanta, 161, 755761,2016

Reijenga, J., Van Hoof, A., Van Loon, A., and Teunissen, B.: Development of methods for the determination of $\mathrm{pKa}$ values, Anal. Chem. Insights, 8, 53-71, 2013.

Robert-Baldo, G. L., Morris, M. J., and Byrne, R. H.: Spectrophotometric determination of seawater $\mathrm{pH}$ using phenol red, Anal. Chem., 57, 2564-2567, 1985.

Safavi, A. and Bagheri, M.: Novel optical pH sensor for high and low pH values, Sensor. Actuat. B-Chem., 90, 143-150, 2003.
Salk, J. E., Youngner, J., and Ward, E. N.: Use of color change of phenol red as the indicator in titrating poliomyelitis virus or its antibody in a tissue-culture system, Am. J. Hyg., 60, 214-30, 1954.

Song, A., Parus, S., and Kopelman, R.: High-performance fiberoptic $\mathrm{pH}$ microsensors for practical physiological measurements using a dual-emission sensitive dye, Anal. Chem., 69, 863-867, 1997.

Stahl, H., Glud, A., Schröder, C. R., Klimant, I., Tengberg, A., and Glud, R. N.: Time-resolved $\mathrm{pH}$ imaging in marine sediments with a luminescent planar optode, Limnol. Oceanogr.-Meth., 4, 336345, 2006.

Tucker, S., Robinson, R., Keane, C., Boff, M., Zenko, M., Batish, S., and Street, K. W.: Colorimetric determination of $\mathrm{pH}$, J. Chem. Educ., 66, 769 pp., 1989.

Weigl, B. H., Holobar, A., Trettnak, W., Klimant, I., Kraus, H., O'Leary, P., and Wolfbeis, O. S.: Optical triple sensor for measuring $\mathrm{pH}$, oxygen and carbon dioxide, J. Biotechnol., 32, 127-138, 1994.

Yacynych, A. M., Twork, J. V., and McGregor, W. C.: Sensors in bioprocess control, Dekker New York, 1990. 